2005-01-01

\title{
Adiabatic behavior of strongly nonlinear internal solitary waves in slope-shelf areas
}

\section{Vlasenko, Vasyl}

http://hdl.handle.net/10026.1/3859

\subsection{9/2004jc002705 \\ Journal of Geophysical Research-Oceans \\ American Geophysical Union (AGU)}

All content in PEARL is protected by copyright law. Author manuscripts are made available in accordance with publisher policies. Please cite only the published version using the details provided on the item record or document. In the absence of an open licence (e.g. Creative Commons), permissions for further reuse of content should be sought from the publisher or author. 


\title{
Adiabatic behavior of strongly nonlinear internal solitary waves in slope-shelf areas
}

\author{
V. Vlasenko \\ School of Earth, Ocean and Environmental Sciences, University of Plymouth, Plymouth, UK
}

L. Ostrovsky

Zel Technologies/NOAA ETL, Boulder, Colorado, USA

\section{K. Hutter}

Institute of Mechanics, Darmstadt University of Technology, Darmstadt, Germany

Received 9 September 2004; revised 7 January 2005; accepted 2 February 2005; published 19 April 2005.

[1] Transformation of large-amplitude internal solitary waves (ISW) propagating over slope-shelf topography is studied theoretically and with the use of the experimental data collected during the Coastal Ocean Probing Experiment (COPE). Taking into account a very strong nonlinearity of observed waves (the ratio of isotherm displacement to their initial depth reached a value of 5), two different approaches were employed for the theoretical investigations of the wave evolution: numerical simulations in the framework of a fully nonlinear nonhydrostatic system of equations and estimations based on a longwave equation derived for a two-layer fluid without any restrictions on the wave amplitude. Special attention is paid to the adiabatic stage of the wave evolution over a gently sloping bottom when the ISW conserves its energy in the course of propagation and preserves the parameters close to a steady solitary wave corresponding to each local depth. Strong ISWs vary adiabatically along the path of propagation until their vertical scale (amplitude) becomes comparable with the total water depth. This adiabatic process typically ends when a soliton reaches its limiting amplitude, after which the breaking process occurs that leads to the generation of turbulence. For a sharp pycnocline, simplified two-layer models are applicable for the study of the shoaling process roughly within the same limits as for steady solitons over a flat bottom. Even for a relatively smooth stratification, some soliton parameters, such as its velocity and the peak particle velocity, can be satisfactorily evaluated from two-layer models.

Citation: Vlasenko, V., L. Ostrovsky, and K. Hutter (2005), Adiabatic behavior of strongly nonlinear internal solitary waves in slopeshelf areas, J. Geophys. Res., 110, C04006, doi:10.1029/2004JC002705.

\section{Introduction}

[2] In situ measurements and remote sensing observations demonstrate an ability of oceanic internal solitary waves (ISW) to propagate for long distances (up to hundreds of kilometers) from their source of generation. Some observational evidences of such long-range propagation can be found, for instance, in [Osborne and Burch, 1980; Apel et al., 1985; Liu, 1988; Brandt et al., 2002] and many other publications. It is a confirmed fact now that in a number of cases, the observed internal waves are strongly nonlinear. Among the corresponding observations are those in the Celtic Sea [Pingree and Mardell, 1985], in the central Bay of Biscay [New and Pingree, 1990], on the Canadian Shelf [Sandstrom and Oakey, 1995], on the Australian northwest shelf [Holloway, 1987], and near the Oregon coast [Stanton and Ostrovsky, 1998; Trevorrow, 1998].

Copyright 2005 by the American Geophysical Union. 0148-0227/05/2004JC002705\$09.00
[3] Most such measurements refer to one fixed point and rarely permit following the evolution of in situ parameters of a soliton train. For relatively weakly nonlinear solibores, such a study was done in the Sulu Sea [Apel et al., 1985]. For strong solitons, the two-point recording of the same train was made near the Oregon coast during September 1995 in the framework of the Coastal Ocean Probing Experiment (COPE). The experimental data from this region were already discussed in detail, for instance, by Stanton and Ostrovsky [1998], Trevorrow [1998], and Kropfli et al. [1999]. Here we use some of their results for the investigation of the dynamics of ISWs shoaling over an inclined bottom topography.

[4] Detailed measurements of ISW characteristics during COPE were performed at two points: (1) from a Floating Instrument Platform (FLIP) located $25 \mathrm{~km}$ offshore at the $150 \mathrm{~m}$ isobath and (2) from a point that was $20 \mathrm{~km}$ closer to the shore, at the $70 \mathrm{~m}$ isobath. Internal wave measurements from these two sites provided the initial data on currents, temperature, salinity, and density during the passage of 

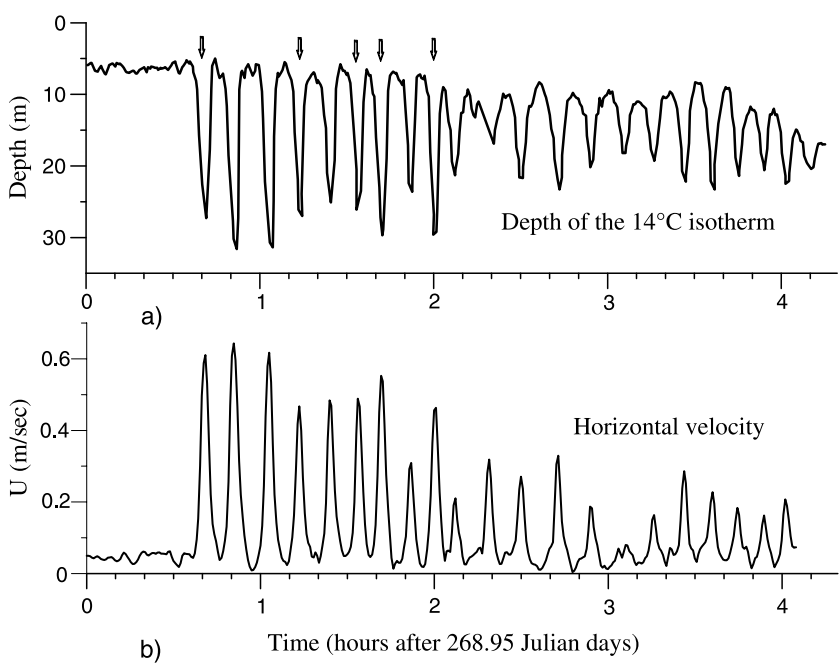

Figure 1. (a) Wave displacements of the $14^{\circ}$ isotherm versus time. (b) Horizontal onshore currents at $8.4 \mathrm{~m}$ depth for the same wave packet. Arrows indicate the waves with amplitudes of about $20 \mathrm{~m}$ used in Figure 8 . The plots are taken from Kropfli et al. [1999].

packets of quasi-plane, strongly nonlinear internal solitary waves (solibores) propagating from the deep part of the ocean toward the shore (for more detailed information, we refer the reader to the papers cited above).

[5] An example of an internal wave measurement reported in the aforementioned papers is presented in Figure 1 [see also Kropfli et al., 1999]. It displays, in particular, a time series of the depth variations for the $14^{\circ}$ isotherm, which is close to the depth of the pycnocline. Figure 1a shows isotherm displacements with amplitudes of $20 \mathrm{~m}$ and greater; such displacements were a common feature of the observed waves. Taking into account also the specific vertical water stratification in the tested region, which is characterized by a very thin (only $5 \mathrm{~m}$ thick) upper mixed layer and a very sharp interface at about $7 \mathrm{~m}$ depth (this can be estimated from Figure 2), this suggests a very strong nonlinearity of the observed waves. Indeed, the ratio of the maximum isotherm displacement to their initial depth for the strongest waves was in a range between 4 and 5 . Moreover, the water velocity at the soliton peaks (Figure 1b) was only slightly smaller than the wave propagation velocity [Kropfli et al., 1999].

[6] The experimental data on characteristics of highly nonlinear internal waves observed during COPE were a strong motivation for us to study the phenomenon and to understand the behavior of a large-amplitude ISW when it propagates over the inclined bottom topography, and which parameters control its dynamics. This situation was relatively well studied only for weakly nonlinear waves when the wave amplitude is small as compared with vertical scales such as the pycnocline depth or the total basin depth.

[7] Several analytical and semianalytical models have been developed and applied to study the shoaling of weakly nonlinear waves [Djordjevic and Redekopp, 1978; Helfrich and Melville, 1986; Holloway et al., 1999]. For largeamplitude ISWs the description of the wave transformation is not so straightforward as it is for weak ones because of the strong nonlinearity of the considered phenomenon. The detailed numerical analysis of the dynamics of strongly nonlinear waves has been performed in the framework of a fully nonlinear nonhydrostatic model in several publications, most recently by Grue et al. [1999, 2000], Vlasenko and Hutter [2001, 2002a, 2002b], and Lamb [2002, 2003]. This is the method that we follow below: we shall consider the evolution of highly nonlinear solitary internal waves over the inclined bottom topography in a situation when the wave amplitudes are comparable with the pycnocline depth or even total water depth.

[8] As distinct from, e.g., Lamb [2002, 2003], we do not consider shoaling of ISWs with a trapped core. Evidence of such waves in the atmosphere was reported, for instance, by Doviak and Christie [1989]. First mode internal solitary waves with recirculation cell were observed in the laboratory experiments by Grue et al. [2000]. There are also some computations of large-amplitude solitary waves with regions of recirculation [see, e.g., Tung et al., 1982; Derzho and Grimshaw, 1997]. In our modeling we also observed stable large-amplitude ISWs with a trapped core.

[9] Recent publications by Voronovich [2003] and Fructus and Grue [2004] should also be mentioned in this connection. A semianalytical approach to the description of strong ISWs was developed in these papers. In the former, steady solitary waves were found in a long-wave limit for a twolayer fluid with each layer having a constant buoyancy frequency. A similar result was obtained for a three-layer fluid by Fructus and Grue [2004] with the use of integral equations and Fourier transform. In both cases a trapped core was included into the solution. However, these analytical approaches are valid only for steady state progressive waves when motion in each layer is described by a linear equation.

[10] Note, however, that no observations of ISWs with trapped core have been made in the ocean so far. Thus we focus on the adiabatic behavior of "ordinary" ISWs taking the parameters of incident waves, as well as the water

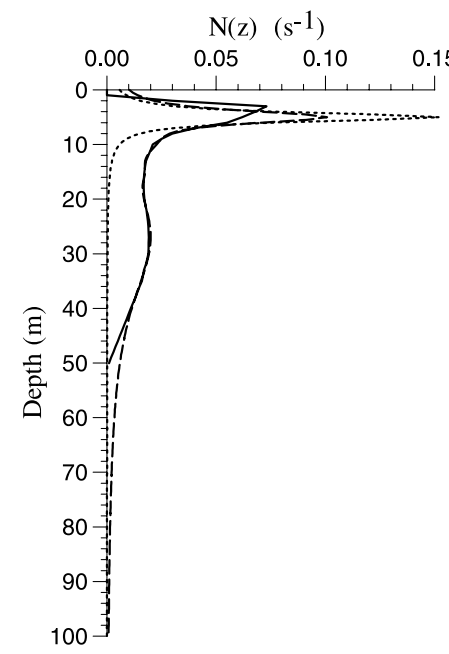

a)

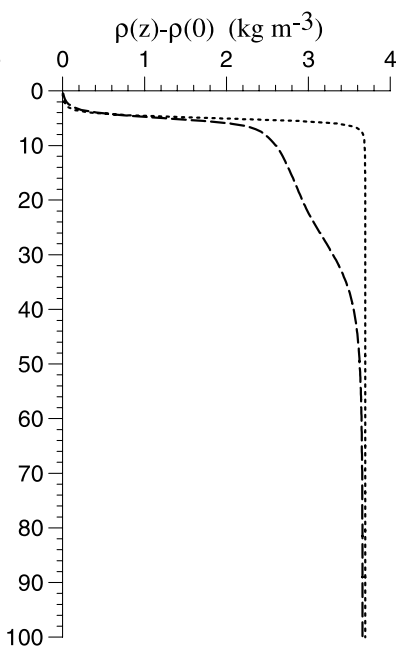

b)
Figure 2. (a) Buoyancy frequency profiles and (b) the appropriate profiles of the density anomaly. The solid line is the curve obtained by smoothing the experimentally measured profile [Trevorrow, 1998]. The dashed line is the profile used in the numerical modeling. The dotted line is the profile used for some estimations. 
stratification and the geometry of the calculation domain close to those observed in the COPE region.

[11] For nonsteady strongly nonlinear waves, semianalytical methods were developed based on a long-wave approximation in papers by Miyata [1988], Choi and Camassa [1999], and Ostrovsky and Grue [2003]. For horizontally homogeneous models such a long-wave theory gives in many cases good agreement with numerical simulations and with the data of laboratory experiments [Michallet and Barthelemy, 1998] and oceanic observations [Ostrovsky and Grue, 2003]. The long-wave approach to strong ISWs in a horizontally inhomogeneous ocean was briefly considered by Tae-Chang and Choi [2002] for a sloping bottom and by Craig et al. [2004] for a sloping pycnocline, both for a two-layer model. However, except for one example given in the former paper, no other solutions were presented and no detailed analysis or comparison with direct computation has been done.

[12] In this paper we study the ISW transformation over a sloping bottom numerically and then apply a simplified two-layer, long-wave model to approximate the same process with the purpose of determining whether and when the simplified approach can satisfactorily work for the realistic example considered here. Observational data are also used for comparison.

[13] The numerical approach that we follow here is similar to that used by Vlasenko and Hutter [2002a, 2002b]. However, unlike these papers, here we do not consider the phenomenon of wave breaking in any detail. Instead, we shall focus on the evolutionary stage of highly nonlinear waves with the aim of discovering how their parameters depend on the shape of the bottom when the internal waves are still far enough from breaking. Note that interaction of strong internal waves with an underwater sill was recently studied by Vlasenko and Hutter [2001, 2002b] for laboratory and lake conditions. In the present study we expand our interest to the oceanic scales and consider shoaling of strong solitary waves in a slope-shelf area. The results show that the adiabatic stage of soliton evolution can last longer than for weakly nonlinear waves and that the simplified two-layer model can describe this process sufficiently well. Experimental data are also used for verifying our calculations.

\section{Formulation and Initialization of Numerical Model}

[14] A fully nonlinear nonhydrostatic numerical model for continuous vertical fluid stratification is used here to study the adiabatic behavior of ISWs over bottom topography. The model was described in earlier papers [Vlasenko and Hutter, 2001, 2002a, 2002b], and here we outline only briefly the basic features of its formulation and initialization.

[15] The two-dimensional system of equations in the Boussinesq approximation, written in Cartesian $x-z$ coordinates in which the $x$ axis coincides with the undisturbed free surface and the $z$ axis is vertical, opposite to the direction of gravity, is used in the form

$$
\begin{aligned}
\omega_{t}+J(\omega, \psi) & =g \rho_{x} / \rho+a_{h} \omega_{x x}, \\
\rho_{t}+J(\rho, \psi) & =k_{h} \rho_{x x}, \\
\omega & =\psi_{x x}+\psi_{z z}
\end{aligned}
$$

for the stream function $\psi(x, z, t)\left(u=\psi_{z}, w=-\psi_{x}\right.$, where $u$, $w$ are the horizontal and vertical velocity components) the vorticity $\omega(x, z, t)$ and the water density $\rho(x, z, t)$. Here $a_{h}$ and $k_{h}$ are, respectively, the coefficients of horizontal viscosity and diffusion, $J(a, b)=a_{x} b_{z}-a_{z} b_{x}$ is the Jacobian operator and the subscripted indices $x, z, t$ denote partial derivatives with respect to the subscripted variable. The system is written for constant coefficients of horizontal eddy viscosity and diffusivity. The coefficients $a_{h}$ and $k_{h}$ were taken to be as small as possible to keep dissipation as small as possible, but still to guarantee stability of the numerical scheme.

[16] We are only interested in baroclinic motions, and thus use the "rigid lid" condition at $z=0$ :

$$
\psi=0, \quad \omega=0, \quad \rho_{z}=0 .
$$

The bottom, $z=-H(x)$, is a streamline at which

$$
\psi=0, \quad \omega=0, \quad \rho_{\mathbf{n}}=0,
$$

where $\mathbf{n}$ is the unit normal vector to the inclined bottom relief.

[17] The choice of $\psi=0$ in (2) and (3) guarantees vanishing of horizontal flux. The boundary conditions for the vorticity are similar to those used for ideal fluid; in our case they are valid with good accuracy, taking into account a zero coefficient of vertical viscosity, $a_{v}$, and a very small value used for $a_{h}$ (see below). In a more general case, when $a_{v} \neq 0$, the no-slip conditions $u=w=0$ (where $u$ and $v$ are horizontal and vertical components of the velocity vector, respectively) must be satisfied at the bottom. In such a case, the value of the vorticity $\omega=\omega_{0}$ at $z=-H(x)$ is usually calculated from the stream function obtained at the previous temporal step. At the free surface, boundary conditions (2) are valid also in a "viscous" case if no tangential stress is applied at $z=0$ (when wind forcing is not considered). For the density we took "zero flux" boundary conditions using the same reasoning.

[18] The problem (1)-(3) was solved numerically with the use of the alternation direction implicit method [Marchuk, 1974]. Before the finite difference implementation, a $\sigma$ transformation of the $z$ coordinate $(\sigma=-z / H(x))$ is performed, which transforms the physical domain with variable depth into a rectangular computational area. This allowed us to use a rectangular grid in the development of a numerical scheme.

[19] The vorticity transport equation is integrated in time by splitting the temporal step into two semisteps. At the first step, the $z$ direction is made implicit, while the $x$ direction is kept explicit. At the subsequent step, the $z$ direction is made explicit, but the $x$ direction is implicit. The spatial derivatives are approximated by second-order central differences. At each temporal semistep a linear system of equations with a tridiagonal matrix is obtained that is solved using standard techniques. The stream function is then computed from the vorticity by solving the Poisson equation $\omega=\psi_{x x}+\psi_{z z}$, previously transformed with the use of the $\sigma$ coordinate. First, this equation is transformed to an equation of evolutional type by adding the temporal derivative, then the stationary solution is sought with the use of the optimal series of the iteration parameters. Finally, the density $\rho$ is 


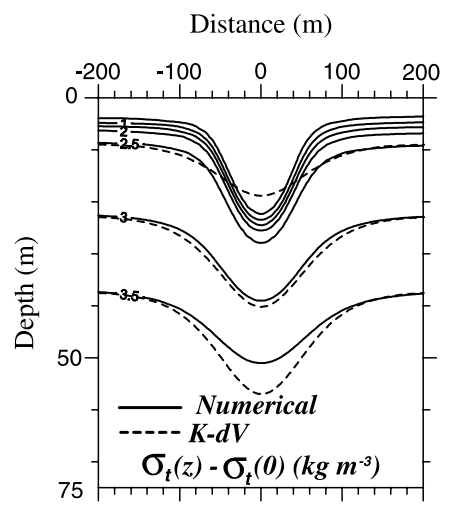

a)

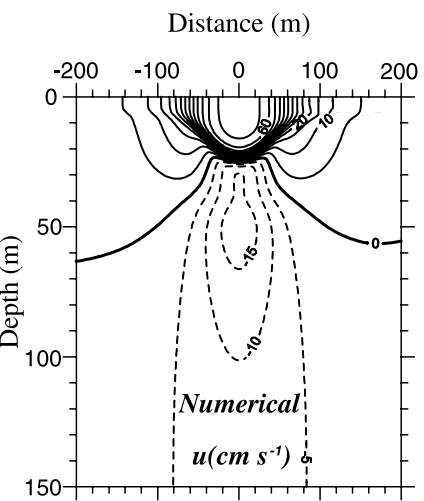

b)

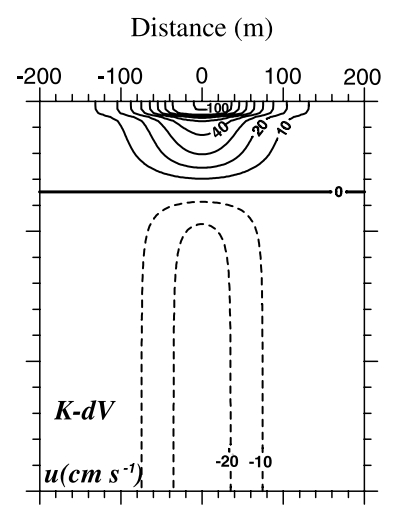

c)

Figure 3. Fields of (a) density anomaly $\left(\mathrm{kg} \mathrm{m}^{-3}\right)$ and $(\mathrm{b}, \mathrm{c})$ horizontal velocity $\left(\mathrm{sm} \mathrm{s}^{-1}\right)$ of $20 \mathrm{~m}$ amplitude internal solitary waves (ISW). Fields in Figures $3 \mathrm{~b}$ and $3 \mathrm{c}$ hold for numerical and K-dV solitary waves, respectively. Solid lines in Figure 3a represent the density field obtained numerically for continuous fluid stratification depicted by the dashed line in Figure 2; the dashed lines show the analogous field for the analytical K-dV solitary wave.

computed by the same methodology as that used for the vorticity transport equation. For more information we refer the reader to the book by Vlasenko et al. [2005], where the numerical scheme is described in greater details.

[20] Several numerical experiments were performed to determine the values of the eddy viscosity and turbulent diffusivity parameters $a_{h}$ and $k_{h}$. It was found that by taking $\Delta t=0.5 \mathrm{~s}, \Delta x=2 \mathrm{~m}, \Delta z=0.5 \mathrm{~m}$ (in the deep part of the basin) and $a_{h}=k_{h}=10^{-4} \mathrm{~m}^{2} \mathrm{~s}^{-1}$, the numerical scheme was stable. These values coincide with measurements and estimates of the background viscosity and diffusivity given by Sandstrom and Oakey [1995]. Such a low level of dissipation allows the internal waves to propagate over a long distance (500 wavelengths and more) without significant attenuation. This is in agreement with in situ observations showing that ISWs can propagate several hundred kilometers from their source of generation [see, e.g., Apel et al., 1985; Brandt et al., 2002].

[21] In accordance with the observations, we assume that a plane solitary internal wave of depression, or a packet of ISWs, propagates from the deep part of the basin to a slopeshelf region. The objective of our modeling efforts was to investigate the process of evolution of strongly nonlinear solitary internal waves over variable bottom topography.

[22] The model was initialized in a similar manner as was done by Vlasenko and Hutter [2002a]. To obtain the initial fields (at $t=0$ ) for the incident wave, at the initial stage we considered a basin with a constant depth of $150 \mathrm{~m}$ (the basin depth at the FLIP location at the COPE site). The corresponding density profile and its approximation used here are shown in Figure 2. For initialization we used the first-mode analytical solitary wave solution of the Korteweg-de Vries (K-dV) equation. Such an initial field represents a stationary solitary wave in a weakly nonlinear case. This K-dV soliton does not satisfy the system (1) for a large-amplitude solitary wave. Thus when being inserted in the numerical scheme, the strong nonlinear wave will evolve in a basin of constant depth until a new stationary solitary wave is formed at the frontal side of the wave field and the leading wave separates from the dispersive wave tail. This wave is then used as an initial condition for the problem of the interaction of intense ISWs with the bottom topography.

[23] We should discuss the properties of such initial solitary waves of finite amplitude and their differences from the K-dV solitons. The structure of one such initial incoming wave with amplitude $20 \mathrm{~m}$ is presented in Figures 3 and 4. Note that large waves differ in detail from the K-dV solitons (we compare two waves, K-dV and numerical, having the same maximum vertical excursion, although at different depths, see Figure 4a). In particular, their vertical structure does not coincide with the eigenfunctions of the linear boundary value problem. In fact, as seen from Figures $3 \mathrm{a}$ and $4 \mathrm{a}$, the wave displacements near the surface are considerably larger in the real wave, but at depth, below the pycnocline, they are much smaller than those for the K$\mathrm{dV}$ case.

[24] Figures $3 \mathrm{~b}$ and $3 \mathrm{c}$ show the horizontal velocity field of the same waves depicted in Figure 3a. The form of the locus of zero horizontal velocity in the numerical wave (Figure $3 b$ ) differs remarkably from the horizontal straight line that results from the K-dV model (Figure 3c). Moreover, the minimum horizontal velocity is not located at the bottom, as in the K-dV solution (compare Figures $3 b$ and $3 \mathrm{c})$. This indicates that large-amplitude oceanic internal solitary waves are much more complicated phenomena than that following from the weakly nonlinear theory. An additional distinction is that the real large-amplitude solitary waves propagate more slowly than the corresponding K-dV waves. Naturally, this discrepancy increases with amplitude growth. For more details on the peculiarities of largeamplitude solitary internal waves, we refer the reader to the papers by Miyata [1985, 1988], Lamb and Yan [1996], Michallet and Barthelemy [1998], Choi and Camassa [1996, 1999], Vlasenko et al. [2000], and Ostrovsky and Grue [2003].

[25] In the present study, the amplitudes $a_{m}$ (maximum displacements of the isopycnals) of the incident internal waves were in a range between 5 and $20 \mathrm{~m}$ (typically observed during COPE, see Figure 1). As mentioned above, 

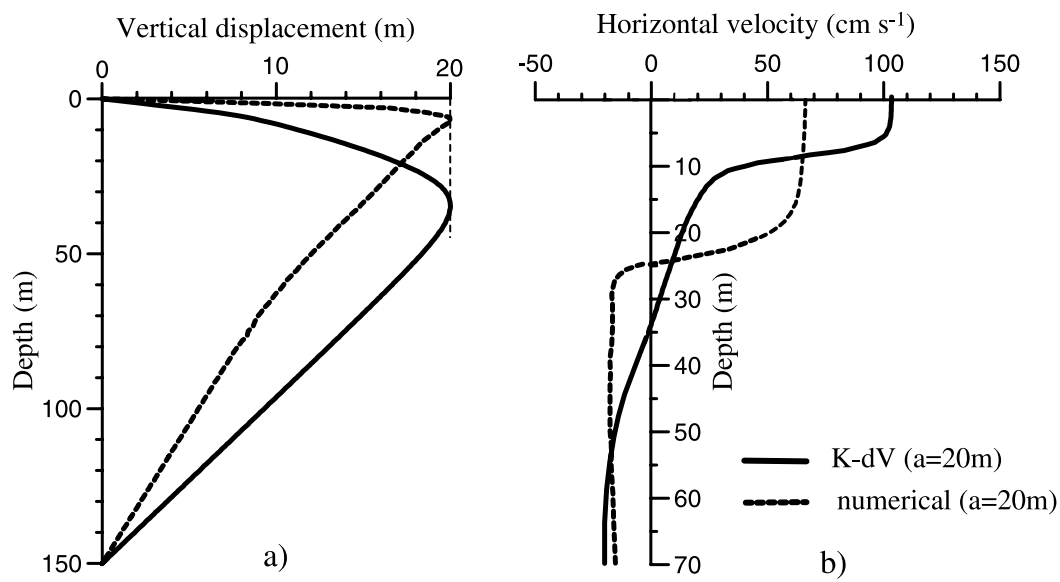

Figure 4. Vertical profiles of (a) isopycnal displacements and (b) horizontal velocity calculated at the wave center for $20 \mathrm{~m}$ amplitude ISW. The numerical and K-dV profiles are given by dashed and solid lines, respectively. (Vertical excursions of the K-dV and numerical waves are equal, although at different depths.)

waves with such amplitudes must be extremely nonlinear: the ratio of the maximum vertical isopycnal displacement to their initial depth varies from 1 to 5 . Figure 5 confirms this statement. The wavelength-amplitude relationship is plotted here for different theoretical models and different density stratifications. The wavelength is defined as follows:

$$
\lambda(z)=\frac{1}{a(z)} \int_{-\infty}^{\infty} \xi(x, z) d x
$$

Here $\xi(x, z)$ represents the displacement of the isopycnals of which the undisturbed depth is $-z$, and $a(z)$ represents its value at the wave center [Miyata, 1988]. In the limit $a \rightarrow 0$, this formula gives the wavelength $\lambda$ for the K-dV solitary wave.

[26] Note that the numerical wavelength decreases first with growing amplitude, as predicted by the K-dV model. For relatively strong waves their width remains almost independent of the amplitude over a wide range of values [Ostrovsky and Grue, 2003], whereas at large amplitudes the wavelength grows again when $a_{\mathrm{m}}$ approaches the maximum amplitude. As seen from Figure 5, the fully nonlinear model indeed reproduces the observed peculiarity [Stanton and Ostrovsky, 1998]: the solitary wave profiles in a range of 7-20 m wave amplitudes are almost identical to each other. The horizontal scale of such waves can be estimated as approximately $100-120 \mathrm{~m}$. The relatively small variation of the wavelength at $a_{m}>10 \mathrm{~m}$ implies that the waves considered are strongly nonlinear.

\section{Comparison With Observational Data}

[27] Before considering the evolution of ISWs over variable bottom topography, let us compare the model results with the available experimental data. We perform this analysis to ascertain that the numerical model correctly reproduces the basic features of strong waves measured at the COPE site.

[28] Figure 6 shows the vertical wave profiles that are similar to those presented in Figure $4 \mathrm{a}$ but for the $70 \mathrm{~m}$ isobath (the second observational point during the COPE).
As reported by Trevorrow [1998], the vertical structure of two strong solitary waves with amplitudes of about 12.5 and $10.8 \mathrm{~m}$ were measured here (the details on the experimental and processing technique can be found therein). The normalized measured vertical displacement profiles are presented in Figure 6 by circles and squares. It is seen that the model-predicted profile (dashed line) computed for ISWs with an amplitude of $12 \mathrm{~m}$ fits the experimental wave profiles much better than the analytical K-dV solution.

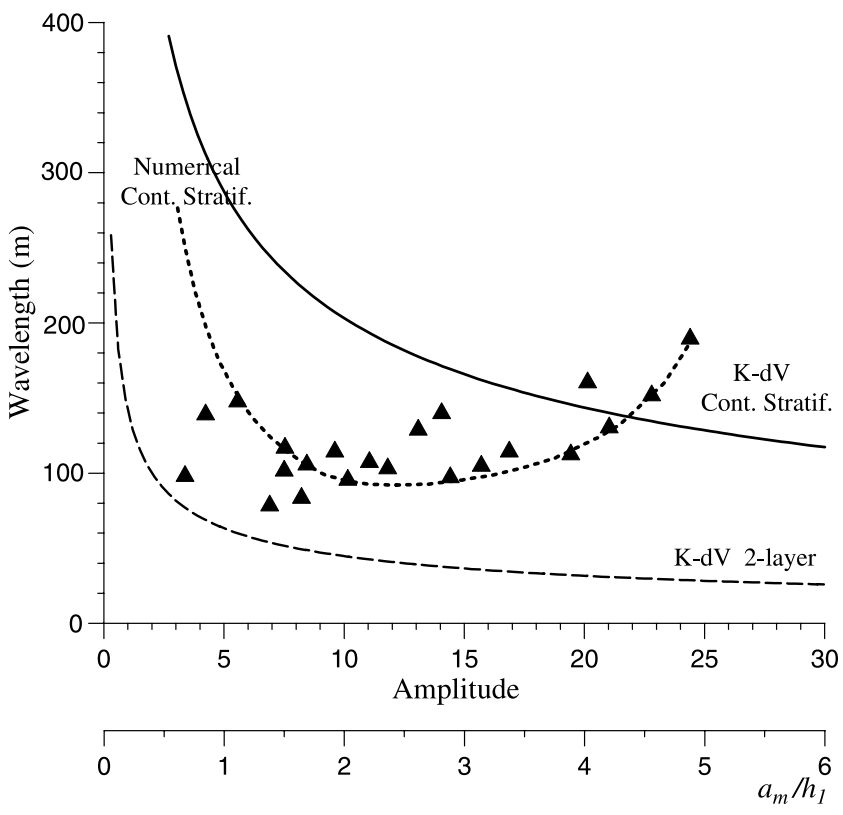

Figure 5. Relationship between wavelength and wave amplitude of internal solitary waves as simulated by the numerical model (dotted line) and as calculated by different models for $H=150 \mathrm{~m}$. Solid and dashed lines were calculated by the first-order K-dV theory for two density profiles presented in Figure $2 \mathrm{~b}$. The abscissa is also shown as the ratio of the wave amplitude to the thickness of the upper layer. Filled triangles are COPE observations [Ostrovsky and Grue, 2003]. 


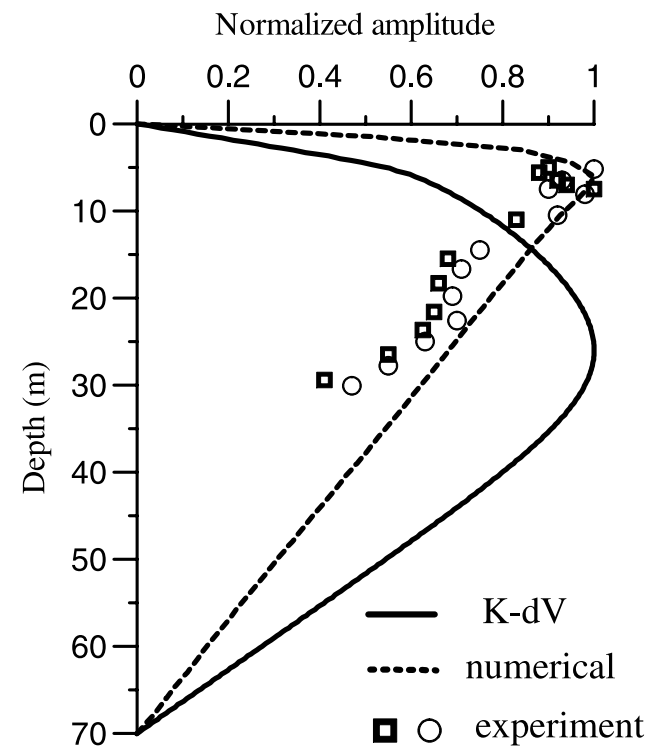

Figure 6. Normalized vertical profiles of isopycnal displacements obtained for the K-dV soliton (solid line) and calculated by means of the fully nonlinear model for a solitary wave with amplitude $a_{\mathrm{m}}=12 \mathrm{~m}$ (dashed line). Two normalized experimental wave profiles measured at the isobath of $70 \mathrm{~m}$ are depicted by the circles and squares [Trevorrow, 1998].

[29] The next test is a comparative analysis of the two time series, presented in Figure 1 with the model results. For every strong depression presented there, we estimated the wave amplitude as the vertical excursion of the $14^{\circ}$ isotherm

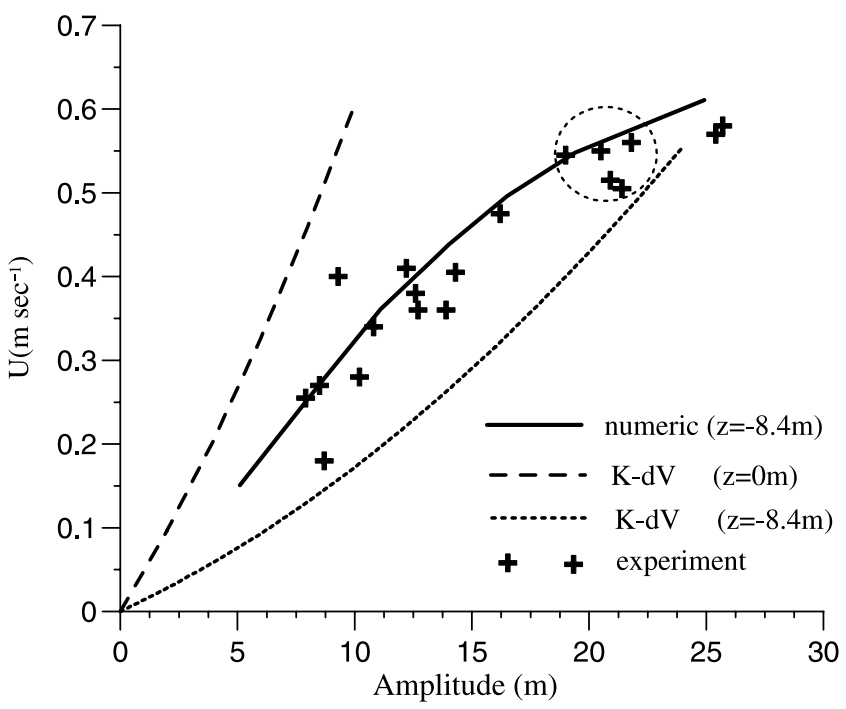

Figure 7. Relationship between the horizontal velocity in the soliton center at $z=-8.4 \mathrm{~m}$ and the wave amplitude as simulated by the numerical model (solid line) and as obtained from in situ data (crosses). For comparison, similar dependencies computed for the $\mathrm{K}-\mathrm{dV}$ solitons are also displayed by the dashed line (at $z=0$ ) and the dotted line (at $z=-8.4 \mathrm{~m})$. For the dashed circle, see discussion in main text. Profile $N(s)$ depicted by the dashed line in Figure 2 is used in the continuously stratified model.

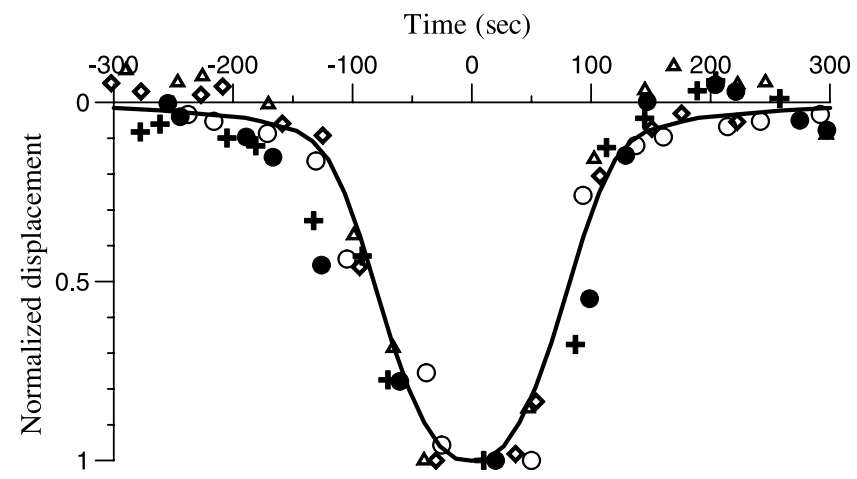

Figure 8. Fit between the normalized isotherm displacements for five solitons marked by arrows in Figure 1. The solid line represents the normalized wave profile obtained from the numerical model for solitary waves with amplitude $a_{\mathrm{m}}=20 \mathrm{~m}$.

shown in Figure 1a and its horizontal velocity at $z=-8.4 \mathrm{~m}$, where the measuring device was located (Figure 1b). As a result we could plot the experimental dependence of the horizontal velocity at the soliton center on wave amplitude. It is represented by crosses in Figure 7 . The analogous dependence, albeit obtained with the use of the fully nonlinear numerical model, is shown in the same figure by the solid line. One can conclude that this curve approximates the cluster of the experimental points much better than do the two other theoretical curves calculated with the $\mathrm{K}-\mathrm{dV}$ theory.

[30] Finally, we compared the shape of every individual solitary wave depicted in Figure 1 with those obtained by the numerical model. We choose for the analysis five solitary waves with comparable amplitudes, and, as expected, equally similar wavelengths $\lambda$ and close phase speeds $V$. These waves are marked by arrows in Figure 1a, and located within the circle in Figure 7 . The result is presented in Figure 8. The experimental points belonging to different waves are represented by different symbols. The theoretical curve for the solitary wave with an amplitude of $20 \mathrm{~m}$ is shown by a solid line. It was calculated from the density field presented in Figure 3a: spatial profile of the isopycne $\sigma(z)-\sigma(0)=2 \mathrm{~kg} \mathrm{~m}^{-3}$, situated just in the middle of the pycnocline, was transformed into the temporal dependence by its division by the wave speed $V$. According to Kropfli et al. [1999], $14^{\circ} \mathrm{C}$ isotherm represented in Figure 1a, also coincides with the middle of the pycnocline.

[31] The basic conclusion that can be drawn from our comparison between the in situ data and the theoretical analysis in general and the results of Figure 8 in particular, is that the fully nonlinear model describes the strong waves observed during COPE quite satisfactorily.

[32] Thus it is hoped that the model can also be used as a tool in our study of the evolution stage of strong solitary waves over the bottom topography, which is carried out below.

\section{Shoaling of Strong Internal Solitary Waves (ISW)}

[33] The bottom topography between the two observational points at the COPE site is characterized by a small 


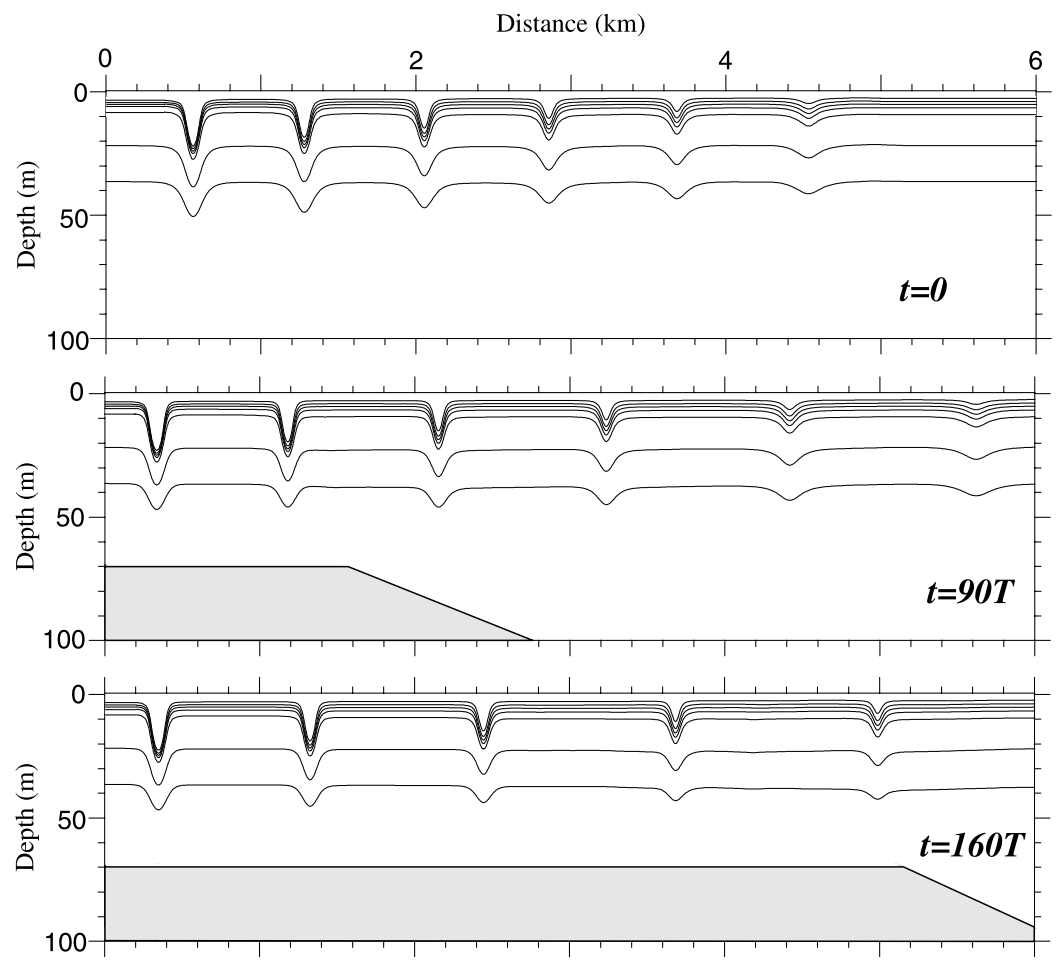

Figure 9. Evolution of six solitary internal waves with amplitudes of $20,17.5,15,12$, 9, and $5 \mathrm{~m}$ penetrating from the deep water region with a depth of $150 \mathrm{~m}$ into the shallow water zone with a depth of $70 \mathrm{~m}$ over the inclined linear bottom topography with inclination angle of $1.47^{\circ}$. The timescale is $T=$ 107 s. The water stratification was close to that observed at the COPE site (dashed line in Figure 2).

inclination angle of about $0.2^{\circ}$. The shoreward water depth varies slowly from $150 \mathrm{~m}$ to $70 \mathrm{~m}$ over a distance of $20 \mathrm{~km}$. As a consequence, the horizontal scale of the bottom variations in the observational area is much larger than the wavelength of the incoming waves. In such a case one can expect a very weak wave reflection from the bottom slope. It is very likely that the propagating waves vary adiabatically along the propagation path until their vertical scale (amplitude) becomes comparable with the maximum possible amplitude in a given point. This assumption must be carefully checked, and this is done in what follows for the COPE site. However, we formulate the problem in this study somewhat more comprehensively than only to obtain a simple explanation of wave dynamics in one observation area. We will try to find the basic parameters that control the adiabatic propagation of solitary waves in a more general case. For this reason we consider not only the bottom profiles inherent to the COPE site, but also profiles with much steeper inclinations.

[34] The idea of the numerical runs is the following: we consider the evolution of solitary internal waves with amplitudes from 5 to $20 \mathrm{~m}$ propagating from the deep part of a basin with a depth of $150 \mathrm{~m}$ to the shallow water zone, where the basin depth $H_{\text {shelf }}$ varies in the range from 40 to $110 \mathrm{~m}$. The bottom profile between the deep and shallow water zones is linear with an inclination angle $\gamma$ of $0.2^{\circ}$, $0.4^{\circ}, 1.5^{\circ}, 6^{\circ}$ and $12^{\circ}$. Our goal is to find out how the characteristics of the waves transmitted to the shallow water zone depend on their amplitudes, on the bottom inclination and water depth. Note that we deal with extremely strong waves in which the nondimensional vertical excursion of fluid particles, i.e., the ratio of the wave amplitude to the initial depth of the undisturbed pycnocline, varies from 1 to 4. Thus some strongly nonlinear effects such as wave breaking can take place during the wave shoaling; this effect was already considered in detail in many papers. The breaking criterion derived from the laboratory experiments by Helfrich and Melville [1986], Helfrich [1992], and Sveen et al. [2002], or from the numerical modeling by Vlasenko and Hutter [2002a], embraces a wide range of bottom inclinations. Here we are not going to particularly study the breaking events; all such cases are deliberately excluded from our analysis. Instead, we will focus on changes of local wave characteristics with the aim of understanding whether and when they obey the law of adiabatic propagation.

[35] First we consider shoaling of an internal wave packet as it was observed at the COPE site. With this example we illustrate a typical situation occurring when the wave train propagates over the bottom topography with relatively small inclination. An example of the wave evolution is presented in Figure 9. In this numerical experiment the initial distance between two adjacent waves at $t=0$ was about $800 \mathrm{~m}$. The incident wave train presented in the upper panel is arranged by amplitude. As can be concluded from the analysis of the two lower panels, none of the solitary waves propagating over the inclined bottom is destroyed and neither do they evolve into dispersive wave trains during propagation. Instead, they adjust permanently to the changing ambient conditions, preserving the shapes and structure inherent to solitary waves. The single evident difference between the wave patterns in the deep part of the basin at $t=0$ and on 

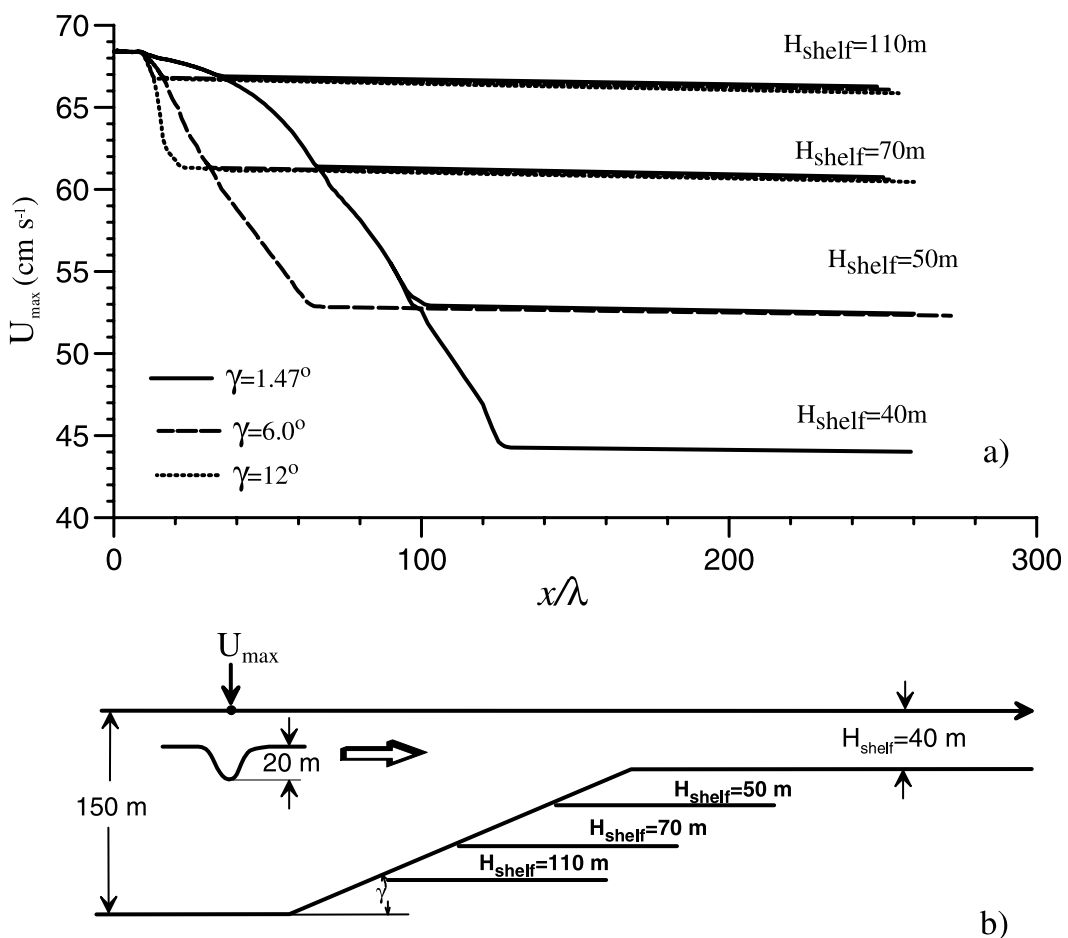

Figure 10. (a) Dependencies of the maximum horizontal velocity (located in the central section of the soliton at the free surface) on the soliton position during its propagation from the deeper part of the basin to the shelf through the linear transition zone. Solid lines correspond to the gently sloping topography with $\gamma=1.47^{\circ}$; dashed and dotted lines have been constructed for edge steepness of $\gamma=6^{\circ}$ and $\gamma=12^{\circ}$, respectively. The water depth on the shelf is indicated in the upper panel for every curve. Only those lines are shown for which the solitary wave was not destroyed. (b) Concept of the numerical experiments. The amplitude of the incoming wave in all cases is $20 \mathrm{~m}$.

the shelf (at $t=160 T$ ) is a substantial stretching of the wave packet which is naturally explained by dispersion of soliton velocity.

[36] In fact, there are two basic concurrent factors defining the wave dispersion in a basin of variable depth. The first one is that the speed of the soliton, $V$, depends on its amplitude, $A$ (nonlinear dispersion; we designate the wave amplitude by $A$ in a two-layer model in contrast to $a_{m}$ used for continuous stratification). Larger solitons propagate faster, which leads to the stretching of the wave packets. On the other hand, the phase speed, $V$, of the shoaling wave decreases when the soliton propagates upward over the inclined bottom. This, in turn, could lead to the temporary compression of the wave train at the stage when the leading soliton has just passed the slope while the trailing one is still approaching the slope. We made such an estimate using the formula (7) given below for the soliton velocity, $V$, in the two-layer model, for the case shown in Figure 9. As seen from Figure 9, the soliton amplitudes do not significantly vary on the slope (unlike the current velocity). For the last, fifth soliton in the group having the amplitude $A=5 \mathrm{~m}$ and remaining in the deep layer with a depth of $150 \mathrm{~m}$, equation (7) gives $V \approx 0.58 \mathrm{~m} \mathrm{~s}^{-1}$. For the leading pulse, with $A=$ $20 \mathrm{~m}$, even after arriving at shallow water of $70 \mathrm{~m}$ depth, the same formula gives $V \approx 0.77 \mathrm{~m} \mathrm{~s}^{-1}$. The latter value still exceeds the maximum velocity of the trailing pulse. Hence nonlinear dispersion prevails, and the group must continue expanding at all stages, which conforms with Figure 9. Note that elongation of the group of COPE solitons upon its onshore propagation is also seen from the comparison of the data for a depth of $150 \mathrm{~m}$ and $70 \mathrm{~m}$ as done by Gorshkov et al. [2004].

[37] In general, preservation of the wave form over variable bottom topography in the family of solitary waves demands permanent change of the quantitative characteristics of the wave field to maintain the permanent balance between the strong nonlinearity and dispersion. An example of such a process is represented in Figure 10. It shows the dependence of the maximum horizontal velocity located at the free surface in the center of ISW upon the local nondimensional coordinate for several bottom angles and several values of the depth in the shallow water zone (as mentioned, the velocity varies more strongly than the soliton amplitude). Analysis of these curves allows the following conclusions: First, the horizontal velocity decreases substantially over the inclined bottom during wave shoaling. Concurrently, the negative value of the velocity, which is located near the bottom, increases (not presented here). However, in the shelf zone, where the depth does not change, the maximum horizontal velocity (and also all other wave characteristics not shown here) is almost constant over a distance of 150-250 wavelengths.

[38] This is an argument in favor of the adiabatic behavior of propagating waves: they adjust permanently to the local depth variations and do not change their characteristics if the depth does not change. The minor decrease of the 
horizontal velocity in the shelf zone (less than $1 \%$ over a distance of 150-250 wavelengths) can be explained by the effect of dissipation which are very weak but still present in the model.

[39] Another fact that confirms the adiabatic behavior of strong ISWs over the inclined bottom is that, in a shelf zone, the curves for different bottom inclinations coincide almost perfectly. Thus we conclude that wave reflection from the inclined bottom by backscattering in the range $\gamma<12^{\circ}$ is almost negligible. However, it does not mean that the wave evolution does not depend at all on the inclination angle $\gamma$. In fact, such a dependence becomes discernable when the solitary wave amplitude becomes comparable with the total water depth; more exactly, the amplitude approaches its maximum value, which in a two-layer fluid is close to $\left(h_{2}-h_{1}\right) / 2$. In such a case the wavelength and the time of its adjustment to the varying bottom topography increases. If the bottom inclination is relatively large and the wave amplitude is close to its possible maximum, the propagating wave does not have enough time for adjustment; as a consequence, it is destroyed near its trailing edge. The mechanism of wave breaking, along with the breaking criterion, were discussed recently by Vlasenko and Hutter [2002a] in detail. In particular, a dependence of the breaking condition on the bottom inclination was found: propagating ISWs are more effectively destroyed over steep bottom features. From the present series of numerical runs it was found that ISWs with an amplitude $a_{m}=20 \mathrm{~m}$ can penetrate into a shelf with $H_{\text {shelf }}=40 \mathrm{~m}$ without breaking if $\gamma<1.5^{\circ}$. The corresponding value for $H_{\text {shelf }}=50 \mathrm{~m}$ equals $6^{\circ}$.

[40] Let us consider now how the other wave characteristics change during wave shoaling. Figure 11 shows the wavelength and wave amplitude dependencies upon the nondimensional time for two waves with amplitudes $a_{m}=$ $20 \mathrm{~m}$ (Figure 11a) and $a_{m}=5 \mathrm{~m}$ (Figure 11b). Evidently, these dependencies are completely different for strong and for weak waves. For strong waves the wavelength increases with an increase of the wave amplitude starting from the moment $t / T$ of about 70 when the wave amplitude begins to "feel" the bottom; this tendency is the opposite for weak waves. Such a behavior is in agreement with the function $\lambda=\lambda\left(a_{m}\right)$ depicted in Figure 5 by the dotted line: the wavelength decreases rapidly with the growth of amplitude for relatively weak waves (when $a_{m}<8 \mathrm{~m}$ ), but it increases for strong waves when $a_{m}>20 \mathrm{~m}$. In the range of $8 \mathrm{~m}<a_{m}<20 \mathrm{~m}$, the wavelength only weakly depends on the amplitude. This is in qualitative agreement with the observational data [Stanton and Ostrovsky, 1998].

[41] The sensitivity of the model results to the angle $\gamma$ of the bottom slope is also seen from Figure 12. The functions $\lambda(x)$ and $a_{m}(x)$ are presented here for waves propagating through the transitional area to the shallow water area with a depth of $H_{\text {shelf }}=70 \mathrm{~m}$. Three different values of the inclination angle $\gamma$ were considered; all other conditions were kept the same. The results also confirm the concept of the adiabatic behavior of the propagating waves. Irrespective of the width of the transitional area (or bottom inclination), the solitary waves in the shallow water zone possess the same characteristics (wavelength and amplitude). The slow decrease of $\lambda$ and $a_{m}$ during propagation can be explained by wave dissipation; we are reminded that the
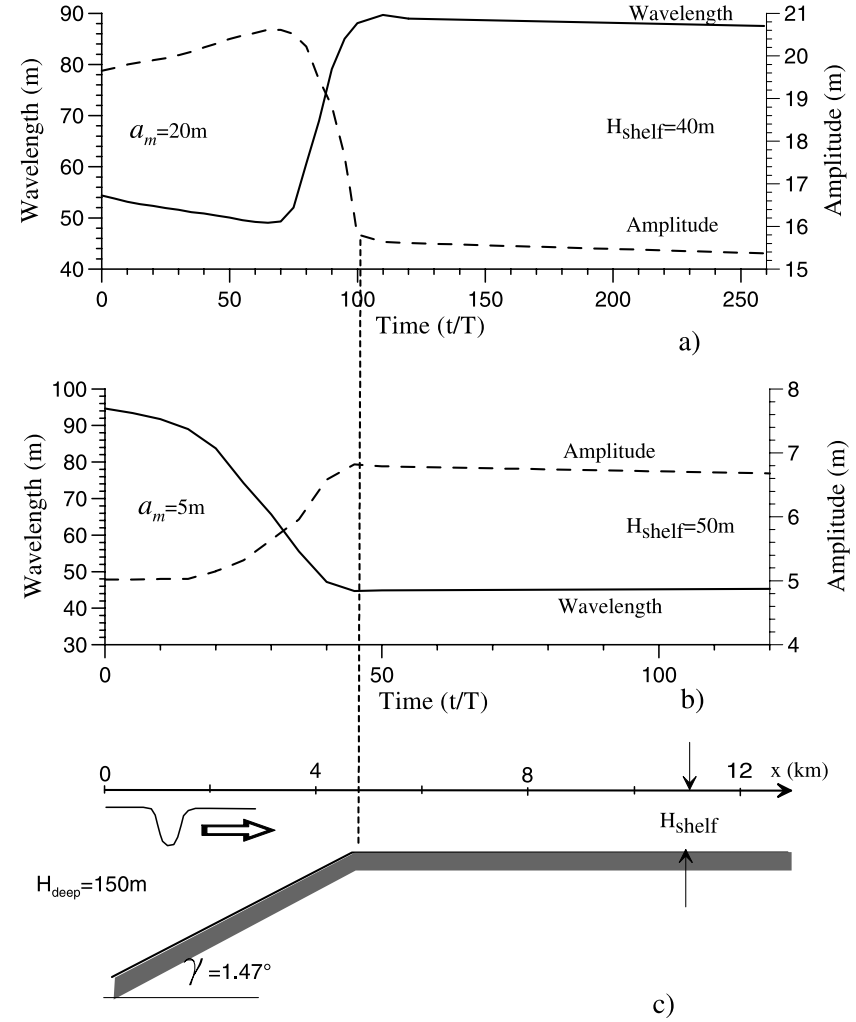

Figure 11. Dependencies of the wavelength (solid lines) and wave amplitude (dashed lines) on the soliton position during its propagation from the deeper part of the basin to the shelf through the linear transition zone. Case of (a) strong solitary waves $\left(a_{\mathrm{m}}=20 \mathrm{~m}\right)$, (b) weak waves $\left(a_{\mathrm{m}}=5 \mathrm{~m}\right)$, and (c) a sketch of the geometry.

length of a strong soliton decreases with the decrease of the amplitude.

\section{Simplified Models for the Evolution of Strong ISW: Comparison With Fully Nonlinear Model}

[42] The results presented above were obtained with the help of a fully nonlinear nonhydrostatic numerical model, developed for a continuously stratified fluid. Its application to concrete oceanic situations by applying a fine-resolution grid (this is the usual requirement for the shoaling process) encounters substantial computational difficulties. However, instead of using a complicated fully nonlinear model, it is often possible to use simpler models that are sufficiently adequate for the analysis of in situ data. In this connection we mention here Miyata [1985, 1988] and Choi and Camassa [1996, 1999] for two-directional equations, and Ostrovsky [1998] and Ostrovsky and Grue [2003] for onedirectional evolution equations. The long-wave models developed in these papers without any restriction on the wave amplitude have been written for a two-layer fluid. Thus their validity for the analysis of real experimental data collected in a continuously stratified ocean depends on the stratification profile and in general it needs verification. Ostrovsky and Grue [2003] used some data of experiments made in the Celtic Sea [Pingree and Mardell, 1985] and 


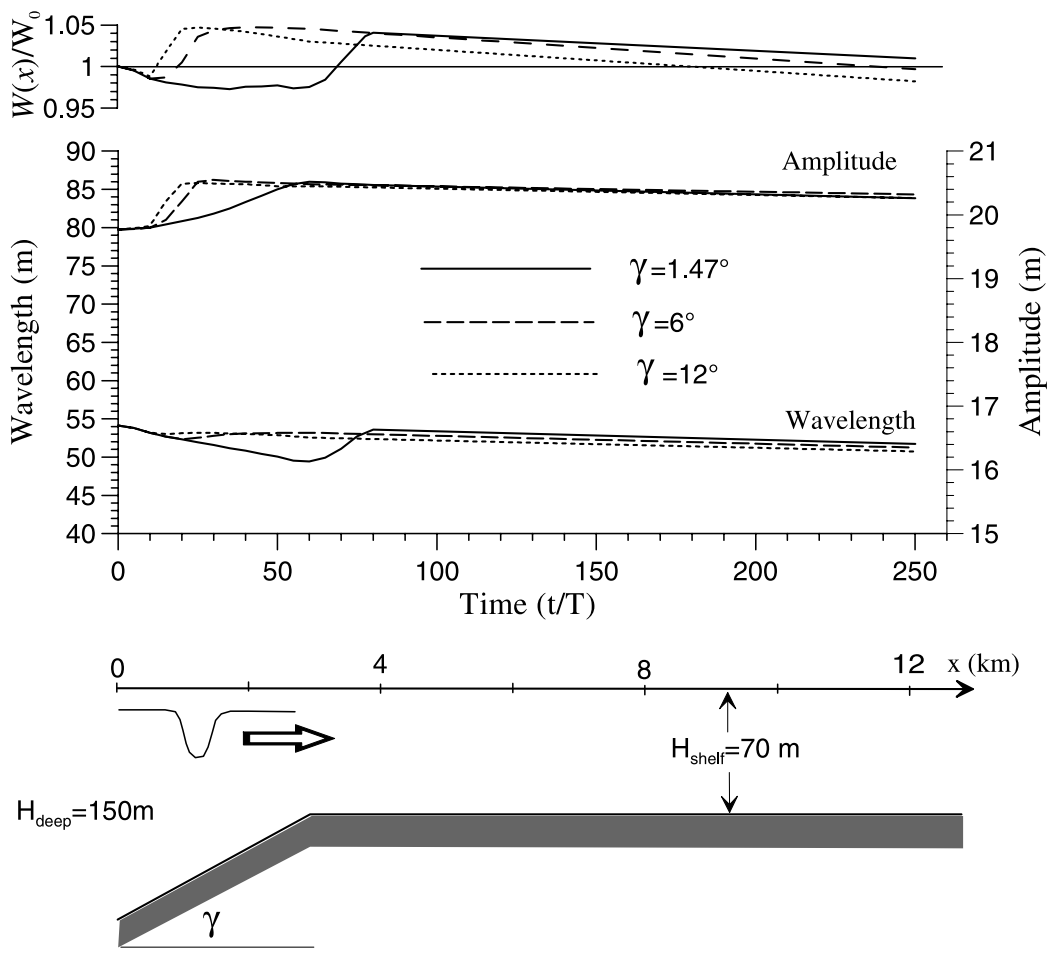

Figure 12. (middle) Same dependencies as in Figure 11, but for different widths (or bottom inclinations) of the transitional area. Designations of all curves are depicted in the figure. The water depth in the shelf area is $70 \mathrm{~m}$ in all cases, and the amplitude of the incident waves is $20 \mathrm{~m}$. (top) Changes of the normalized function (9) across the shelf.

during COPE (considered above) to show that in some cases the agreement between the two-layer models and observations is rather good (especially regarding the soliton speed and horizontal particle velocity); in other cases the twolayer theory is apparently insufficient to describe correctly soliton profiles. Below we compare the results from the two approaches in the application to the COPE site by taking into account both more realistic smooth stratification and propagation over variable depth.

[43] We consider the adiabatic variations of a soliton based on conservation of the total soliton energy, $W$, in a nondissipative system and in the absence of reflections. Here we shall use the expression for the energy density, $E$, per unit length (i.e., integrated over depth) in a long wave, in the form [Ostrovsky and Grue, 2003]

$$
2 E / \rho=\left(h_{1}+\eta\right) u_{1}^{2}+\left(h_{2}-\eta\right) u_{2}^{2}+g^{\prime}\left(\eta^{2}+h_{1} \eta\right) .
$$

Here the subscripts 1 and 2 refer to the upper and lower layer, respectively, $h_{1}$ and $h_{2}$ are unperturbed layer thicknesses, $u_{1}$ and $u_{2}$ are particle velocities averaged over each layer, and $g^{\prime}=g\left(\rho_{2}-\rho_{1}\right) / \rho_{1}$; it is supposed that the density difference is small which is always the case for the ocean. Note that the last term in (5) $g^{\prime} h_{1} \eta$, is unnecessary here due to the independent mass conservation. In Ostrovsky and Grue [2003], it was retained in order to consider an evolution equation (e-model) in which the mass is not exactly conserved. In any case this term does not affect the result (approximate constancy of soliton amplitude).

[44] In Ostrovsky and Grue [2003] an energy-conserving evolution equation (e-model) was suggested, and the rela- tions between $u_{1,2}$ and $\eta$ were taken from the Riemann invariants for a long (nondispersive) wave. Here, for simplicity, we shall consider a single, locally steady solitary wave when all variables are functions of one argument, $\zeta=$ $x-V t$. Then the relationships between the variables follow directly from the mass conservation in the reference frame moving at velocity $V$ where no time dependence exists. For a long wave, when the current in each layer is almost horizontal, we approximately have (this is exact for the peak of the soliton, $\eta=A$ )

$$
u_{1}=\frac{V \eta}{h_{1}+\eta} \quad u_{2}=-\frac{V \eta}{h_{2}-\eta} .
$$

As shown by Ostrovsky and Grue [2003], the soliton velocity in practically all long-wave models considered there as well as from the fully nonlinear calculations, can be represented by the formula

$$
V=\sqrt{\frac{g^{\prime}\left(h_{1}+A\right)\left(h_{2}-A\right)}{H}},
$$

where $H=h_{1}+h_{2}$ is the total local depth on the sea (note that it coincides with the velocity of a linear nondispersive wave if the interface is shifted to the depth $\left.h_{1}+A\right)$. Thus, the energy density (5) at the soliton peak is

$$
E_{\max }=\rho g^{\prime}\left(A^{2}+h_{1} A\right) .
$$

[45] Let us first compare the simple two-layer formulae (6) and (7) with the above numerical results obtained for 


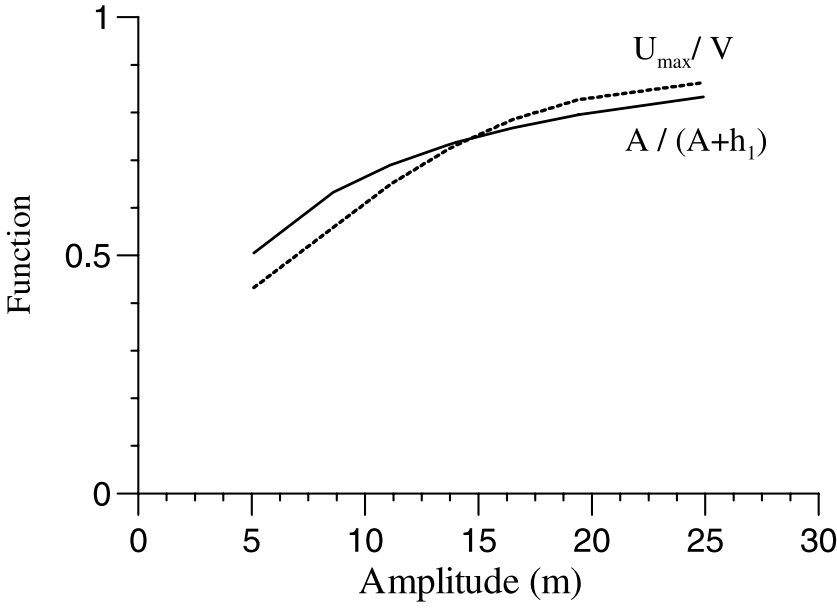

Figure 13. Numerically found dependence of maximum velocity of fluid particles at the free surface in the soliton center, $U_{\max }$, normalized by the nonlinear phase speed $V$, plotted against the wave amplitude (dashed line). The solid line represents the function $A /\left(A+h_{1}\right)$ found from the mass conservation in the two-layer model.

smooth stratification. Here we take $h_{1}=5 \mathrm{~m}$ (the depth of the buoyancy frequency peak in Figure 2). Figure 13 shows the corresponding results for the maximum particle velocity in the upper layer. It is seen that the expression (6) written for the two-layer model and maximum of normalized horizontal velocity $U_{\max } / V$ calculated from the continuously stratified numerical models agree rather well in a wide range of soliton amplitudes.

[46] Figure 14 gives a comparison of present numerical results for soliton velocity at different depths with formula (7) as well as with the corresponding K-dV models. It is interesting to note that whereas the two-layer $\mathrm{K}-\mathrm{dV}$ model gives unrealistic results (as already mentioned by Ostrovsky and Grue [2003]), the K-dV model for the real, continuous stratification, can be closer to reality, especially for a smaller total depth (Figure 14c). At the same time, the two-layer expression (7) works well for a larger depth (Figure 14a) and gives a larger discrepancy for a shallower layer (Figure 14c). This is understandable; indeed, the relative role of a stratified area existing below the pycnocline (Figure 1) should increase with the decrease of the lower layer thickness. Still the discrepancy is moderate even in Figure 14c (never exceeds 20\%), so that we shall use (7) in subsequent estimates.

[47] Consider now wave evolution over the variable depth based on equation (8). In our case $h_{1}=$ const but $h_{2}=h_{2}(x)$, and the bottom slope is again supposedly sufficiently small to be able to neglect wave reflections. In this case the full soliton energy, $W=\int_{-\infty}^{\infty} E d x$, where $E$ is given by (8), must be conserved. To avoid cumbersome calculations, we introduce a characteristic soliton width, $L$, defined at the level of half-amplitude, and estimate $W$ as $E_{\max } L$ (note that $L$ is defined here differently from (4), although these two values are close). To estimate the width $L$ of a soliton, we use the result of the calculation shown by Ostrovsky and Grue [2003, Figure 7]. It shows that in the range of ratios $A / h_{1}$ between 1 and 4 which has been used above for numerical calculations, the dependence $L(A) / h_{1}$ is practically the same for depths of $150 \mathrm{~m}$ to $70 \mathrm{~m}$. Moreover, as seen from Figure 4 of the cited paper, even for a nonCOPE example, when $h_{2} / h_{1}=3.6$, which in our case would correspond to $h_{2}=18 \mathrm{~m}$, the relative width at $A / h_{1}=1$ is about the same as in Figure 7 , namely, $L / h_{1} \approx 12-13$. Note that these curves strongly diverge only when $A$ comes close to its critical value, $A_{\mathrm{cr}}=\left(h_{2}-h_{1}\right) / 2$.
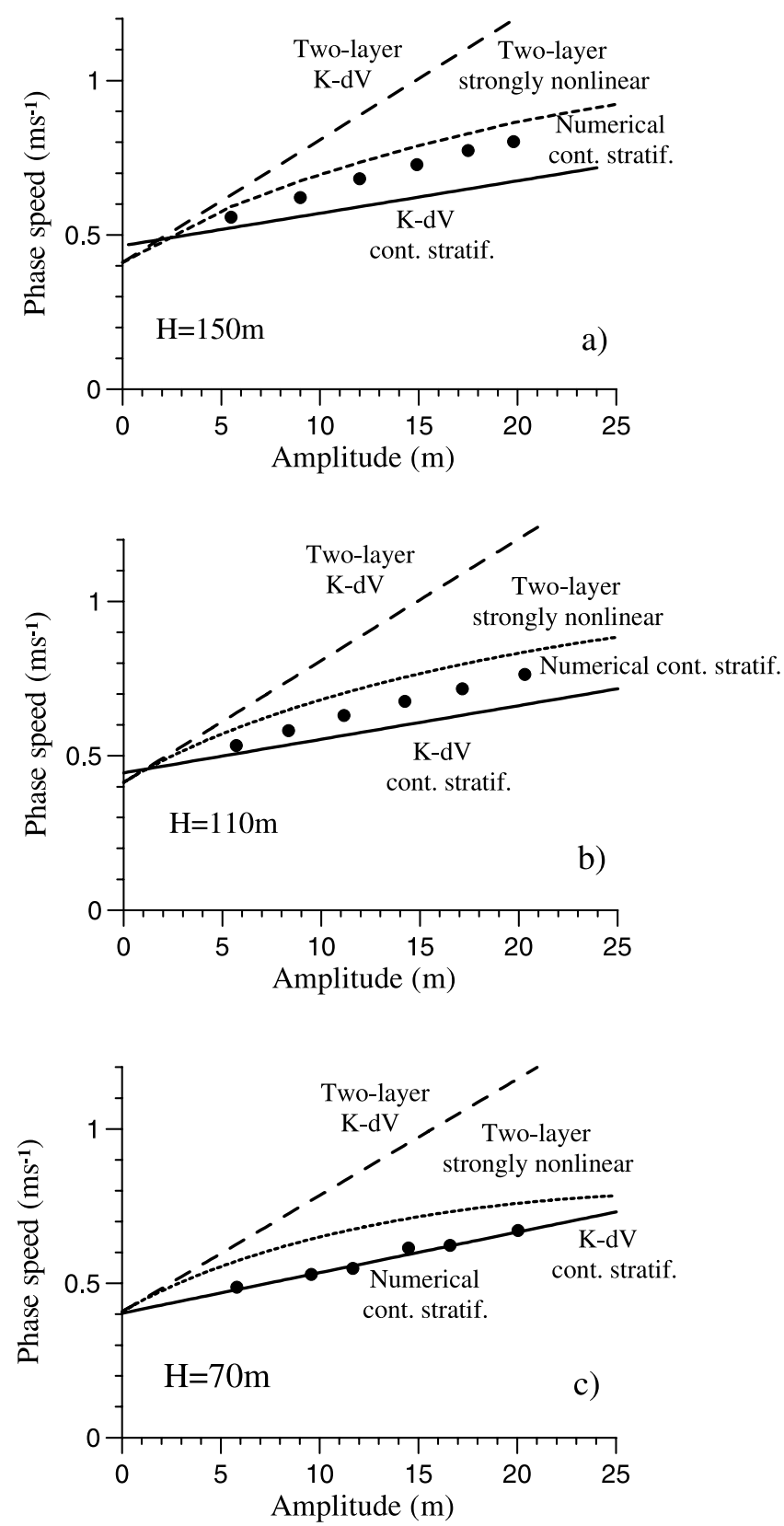

Figure 14. Phase speed of ISWs versus amplitude for different models and different values of water depth at the shelf: (a) $150 \mathrm{~m}$; (b) $110 \mathrm{~m}$; and (c) $70 \mathrm{~m}$. Solid and dashed lines correspond to the continuously stratified and two-layer $\mathrm{K}-\mathrm{dV}$ models, respectively; the dotted line is built according to the two-layer, strongly nonlinear model. Filled circles represent numerical results. Profile $N(s)$ depicted by the dashed line in Figure 2 is used for the continuously stratified models. 


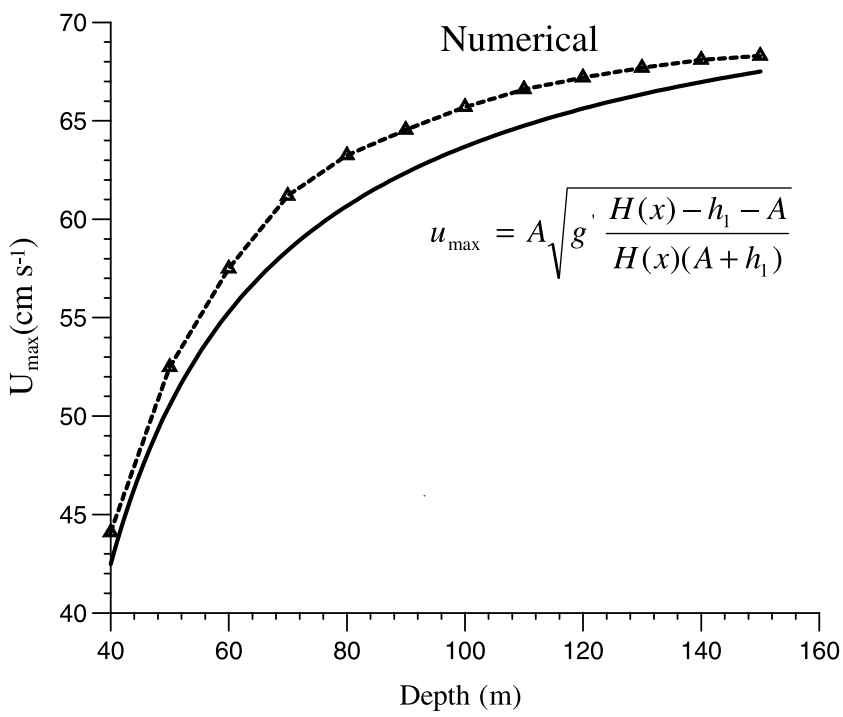

Figure 15. Numerically found dependence of maximum velocity of fluid particles at the free surface at the soliton centers, $U_{\max }$, on water depth (dashed line). The solid line is the function $u_{1 m}(H)$ found from the two-layer model (see equation (10).

[48] Hence in the amplitude range considered here, $L / h_{1}$ is a function of $A / h_{1}$ or, at a constant $h_{1}$, just $L=L(A)$, and this is practically independent of $h_{2}$. As a result we have

$$
W(A) \propto A\left(A+h_{1}\right) L(A)=\text { const. }
$$

From here it follows that in the approximation considered, the soliton amplitude remains constant upon propagation over a sloping bottom. This result may not be precise because the solitary solution is not exactly self-similar, and the energy integral can depend on its specific profile. To verify it, the value of soliton energy $W$ was calculated with the use of (9) where $A$ was taken from the above smooth stratification model; it is shown in the upper panel of Figure 12. From that, one can conclude that in the considered case, the energy of propagating ISW is conserved, within 5\% accuracy, in the framework of the two-layer model.

[49] Also, Figure 12 confirms that even for smooth stratification, the amplitudes of strong solitons vary only slightly in the course of propagation, which confirms the above result.

[50] At the same time, the magnitude of the particle velocity varies significantly more strongly. Indeed, expressing the maximum particle velocity in the upper layer (see (6)) in terms of the total variable depth, $H(x)=h_{1}+h_{2}(x)$ we have

$$
u_{1 \max }=\frac{V A}{A+h_{1}}=A \sqrt{g^{\prime} \frac{\left(H(x)-h_{1}-A\right)}{H(x)\left(A+h_{1}\right)}} .
$$

[51] As already mentioned, the first of these expressions, $\left(A /\left(A+h_{1}\right)\right)$, agrees well with the normalized horizontal velocity, $U_{\max } / V$, calculated by the continuously stratified numerical models (Figure 13). The last expression in (10) represents the dependence of the maximum horizontal velocity on the water depth in the two-layer model at constant $A$. For a shelf with a slope of $1.47^{\circ}$, it is shown in Figure 15 along with the corresponding numerical result for continuous stratification. It can be concluded that estimates with the two-layer formula (10) are quite satisfactory.

[52] Thus the long-wave, two-layer approximation, albeit having limited applicability, satisfactorily describes the main characteristics of the real processes.

[53] A specific case of large ISW behavior occurs when at some point the soliton approaches the critical amplitude, $A_{\text {cr }}(x)=\left(h_{2}-h_{1}\right) / 2$. As already mentioned, in this case the conclusion that soliton length is independent of $h_{2}(x)$ obviously fails. Different scenarios of soliton evolution are in principle possible for ISW approaching the critical amplitude. The first, realized at relatively steep bottom slopes, is wave breaking with generation of turbulence, as was described by Vlasenko and Hutter [2002a, 2002b]. Conditions of wave overturning are described by the breaking criterion in terms of the bottom inclination and wave amplitude.

[54] The second scenario is an adiabatic transformation when the soliton amplitude close to $A_{\text {cr }}(x)$, follows the depth variation so that its length varies to conserve the total energy. This scenario takes place at very low values of the bottom inclination (of order 1 degree and less). Substituting the above expression for $A_{\mathrm{cr}}$ into the total energy (9), we see that the energy of a limiting soliton is proportional to

$$
W_{\mathrm{cr}}(x) \propto\left(A_{\mathrm{cr}}^{2}+h_{1} A_{\mathrm{cr}}\right) L_{\mathrm{cr}} \propto L_{\mathrm{cr}}\left(h_{2}^{2}(x)-h_{1}^{2}\right)=\text { const. }
$$

[55] The corresponding dependence of $L_{\mathrm{cr}}$ on the total depth $H$, normalized by its value at $H=40 \mathrm{~m}$ is shown in Figure 16. As expected, the soliton should expand upon moving onshore (toward smaller depths). This agrees, at least qualitatively, with the numerical results.

[56] Between these two extreme cases, wave breaking and adiabatic adjustment, there also exists an intermediate scenario of the ISW evolution, which can be called "dispersive." In this regime a propagating solitary wave is not destroyed (the wave is far from breaking conditions), but it also does not exactly conserve the energy in the course of propagation. If the breaking criterion is not satisfied but the ISW does not have enough time for the complete adjustment to the features of the relatively steep bottom, it radiates a small portion of energy in the form of a dispersive wave "tail," as shown in Figure 17. Note that up to the time moment $t=t_{0}$ when the ISW reaches the isobath $H=55 \mathrm{~m}$,

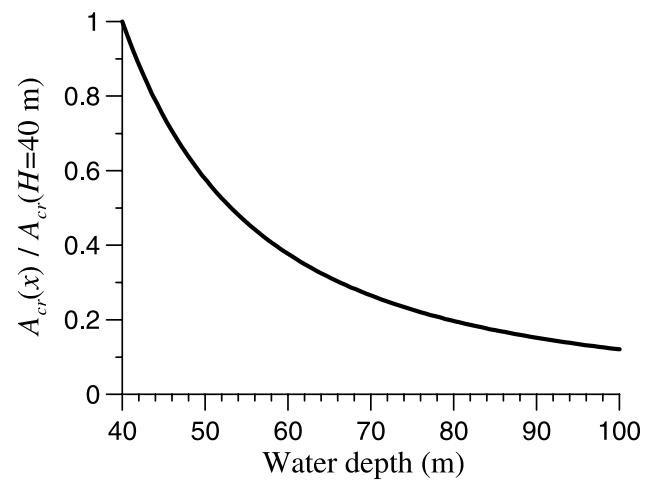

Figure 16. Dependence of the critical amplitude of ISW normalized by its value at $H=40 \mathrm{~m}$ on the water depth. 


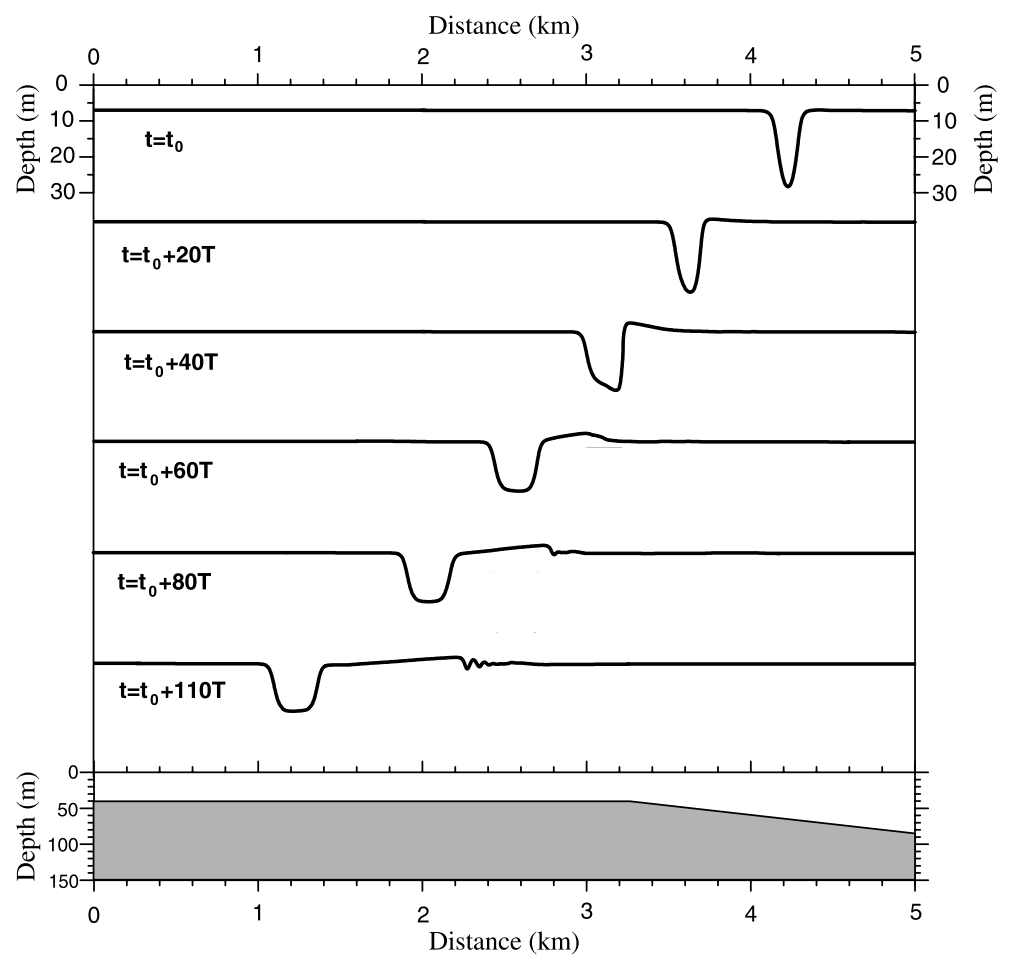

Figure 17. Example of nonadiabatic evolution of the ISW over the gently sloping $\left(\gamma=1.47^{\circ}\right)$ bottom (only the isopycne corresponding to the middle of the density jump is shown). The timescale $T$ equals $45 \mathrm{~s}$. The initial amplitude of the incident ISW beyond the shelf at $H=150 \mathrm{~m}$ is $22.5 \mathrm{~m}$.

it propagates adiabatically, adjusting its profile. Closer to the shore, at $H<50 \mathrm{~m}$, the ISW radiates a small secondary wave packet. Note that this radiation does not significantly change the soliton energy and does not prevent the formation of a critical soliton at the frontal side of the wave field. The amplitude of the limiting ISW in the lower plot can be estimated as $12.8 \mathrm{~m}$, which is very close to the value $A_{\mathrm{cr}}=$ $13.0 \mathrm{~m}$, found from the two-layer model.

\section{Conclusions}

[57] 1. As demonstrated in this paper, strongly nonlinear solitary waves can be adiabatically transformed when propagating over a sloped bottom. In other words, a soliton adjusts the parameters to those of a constant depth that it locally experiences. This conclusion is valid for the majority of realistic bottom inclinations considered in the present paper. Moreover, strong solitons are somewhat more stable in this respect than weakly nonlinear ones, because the nonlinear steepening and dispersive broadening that are balanced in a soliton are stronger factors when compared with smooth bottom variations which, in this sense, turn to be more significant for weak waves than for strong ones. This adiabatic process typically ends after a soliton amplitude approaches its limiting value, after which the adiabatic evolution may not continue, and finally the breaking process occurs that leads to the generation of turbulence.

[58] 2. Another problem considered here is whether the simpler, long-wave, two-layer models can be applicable to describe the adiabatic variations of solitons. In this connection, we derived the corresponding adiabatic formulas based on soliton energy conservation, and compared them with direct numerical simulation. The preliminary result is that for a sharp pycnocline, these models are applicable roughly within the same limits as for the steady solitons considered earlier. Even for a relatively smooth stratification, soliton parameters such as its propagation speed and the peak particle velocity can be satisfactorily evaluated from the simplified models.

[59] Acknowledgments. This work was financially supported by the Natural Environmental Research Council under grant NE/C50747X/1, by INTAS under grant 03-51-3728, and by the Deutsche Forschungsgemeinschaft through grant Hu412/32-1. Comments of anonymous reviewers were most useful in clarifying the presentation.

\section{References}

Apel, J. R., J. R. Holbrock, A. K. Liu, and J. J. Tsai (1985), The Sulu Sea internal soliton experiment, J. Phys. Oceanogr., 15, 1625-1651.

Brandt, P., A. Rubino, and J. Fischer (2002), Large-amplitude internal solitary waves in the North Equatorial Counter Current, J. Phys. Oceanogr., 32, 1567-1573.

Choi, W., and R. Camassa (1996), Long internal waves of finite amplitude, Phys. Rev. Lett., 77, 1759-1783.

Choi, W., and R. Camassa (1999), Fully nonlinear internal waves in a twofluid system, J. Fluid Mech., 396, 1-31.

Craig, W., P. Guyenne, and H. Kalish (2004), A new model for large amplitude long internal waves, C. R. Mecanique, 332, 525-530.

Derzho, O. G., and R. Grimshaw (1997), Solitary waves with a vortex core in a shallow layer of stratified fluid, Phys. Fluid, 9, 3378-3385.

Djordjevic, V. D., and L. G. Redekopp (1978), The fissional disintegration of internal solitary waves moving over two-dimensional topography, J. Phys. Oceanogr., 8, 1016-1024.

Doviak, R. J., and D. R. Christie (1989), Thunderstorm-generated solitary waves: A wind shear hazard, J. Aircraft, 26, 423-431.

Fructus, D., and J. Grue (2004), Fully nonlinear solitary waves in a layered stratified fluid, J. Fluid Mech, 505, 323-347.

Gorshkov, K. A., L. A. Ostrovsky, I. A. Soustova, and V. G. Irisov (2004), Perturbation theory for kinks and its application for multisoliton interaction in hydrodynamics, Phys. Rev. E, 69, 01664. 
Grue, J., A. Jensen, P.-O. Rusas, and J. K. Sveen (1999), Properties of large-amplitude internal waves, J. Fluid Mech., 380, 257-278.

Grue, J., A. Jensen, P.-O. Rusas, and J. K. Sveen (2000), Breaking and broadening of internal solitary waves, J. Fluid Mech., 413, 181-217.

Helfrich, K. (1992), Internal solitary wave breaking and run-up on a uniform slope, J. Fluid Mech., 243, 133-154

Helfrich, K., and W. K. Melville (1986), On nonlinear internal waves over slope-shelf topography, J. Fluid Mech., 167, 285-308.

Holloway, P. E. (1987), Internal hydraulic jumps and solitons at a shelf break region on the Australian north west shelf, J. Geophys. Res., 92, $5405-5416$

Holloway, P. E., E. Pelinovsky, and T. Talipova (1999), A generalized Korteweg-de Vries model of internal tide transformation in the coastal zone, J. Geophys. Res., 104, 18,333-18,350.

Kropfli, R. A., L. A. Ostrovski, T. P. Stanton, E. A. Skirta, A. N. Keane, and V. Irisov (1999), Relationships between strong internal waves in the coastal zone and their radar and radiometric signatures, J. Geophys. Res., 104, 3133-3148.

Lamb, K. (2002), A numerical investigation of solitary internal waves with trapped cores formed via shoaling, J. Fluid Mech., 451, 109-144.

Lamb, K. (2003), Shoaling solitary internal waves: On a criterion for the formation of waves with trapped cores, J. Fluid Mech., 478, 81-100.

Lamb, K. G, and L. Yan (1996), The evolution of internal wave undular bores: Comparison of fully nonlinear numerical model with weakly nonlinear theory, J. Phys. Oceanogr., 26, 2712-2734.

Liu, A. K. (1988), Analysis of nonlinear internal waves in the New York bight, J. Geophys. Res., 93, 12,317-12,329.

Marchuk, G. I. (1974), Numerical Methods in Weather Prediction, 277 pp., Elsevier, New York.

Michallet, H., and E. Barthelemy (1998), Experimental study of solitary waves, J. Fluid Mech., 366, 159-177.

Miyata, M. (1985), An internal solitary wave of large amplitude, La Mer, $23,43-48$

Miyata, M. (1988), Long internal waves of large amplitude, in Nonlinear Water Waves, edited by K. Horikawa and H. Maruo, pp. 399-406, Springer, New York.

New, A. L., and R. D. Pingree (1990), Large-amplitude internal soliton packets in the central bay of Biscay, Deep Sea Res., 37, 513-524

Osborne, A. R., and T. I. Burch (1980), Internal solitons in the Andaman Sea, Nature, 208, 451-469.

Ostrovsky, L. A. (1998), How to describe strong internal waves in coastal areas?, in The 1998 WHOI/ONR Internal Solitary Wave Workshop: Contributed Papers, edited by T. Duda and D. Farmer, Tech. Rep. WHOI-9907, pp. 224-229, Woods Hole Oceanogr. Inst., Woods Hole, Mass.
Ostrovsky, L. A., and J. Grue (2003), Evolution equations for strongly nonlinear internal waves, Phys. Fluids, 15, 293.

Pingree, R. D., and G. T. Mardell (1985), Solitary internal waves in the Celtic Sea, Prog. Oceanogr., 14, 431-441.

Sandstrom, H., and N. S. Oakey (1995), Dissipation in internal tides and solitary waves, J. Phys. Oceanogr., 25, 604-614

Stanton, T. P., and L. A. Ostrovsky (1998), Observations of highly nonlinear internal solitons over the continental shelf, Geophys. Res. Lett., 25, $2695-2698$

Sveen, J. K., Y. Guo, P. A. Davies, and J. Grue (2002), On the breaking of internal solitary waves at a ridge, J. Fluid Mech., 469, 161-188.

Tae-Chang, J., and W. Choi (2002), Dynamics of strongly nonlinear internal solitary waves in shallow water, Stud. Appl. Math., 109, 205-227.

Trevorrow, M. V. (1998), Observations of internal solitary waves near the Oregon coast with an inverted echo sounder, J. Geophys. Res., 103, $7671-7680$.

Tung, K.-K., T. F. Chan, and T. Kubota (1982), Large amplitude internal waves of permanent form, Stud. Appl. Math., 66, 1-44.

Vlasenko, V., and K. Hutter (2001), Generation of second mode solitary waves by the interaction of a first mode soliton with sill, Nonlinear Processes Geophys., 8, 223-239.

Vlasenko, V., and K. Hutter (2002a), Numerical experiments on the breaking of solitary internal waves over slope-shelf topography, J. Phys. Oceanogr., 32, 1779-1793.

Vlasenko, V., and K. Hutter (2002b), Transformation and disintegration of strongly nonlinear internal waves by topography in stratified lakes, Ann. Geophys., 20, 2087-2103.

Vlasenko, V. I., P. Brandt, and A. Rubino (2000), On the structure of large amplitude internal solitary waves, J. Phys. Oceanogr., 30, 2172-2185.

Vlasenko, V., N. Stashchuk, and K. Hutter (2005), Baroclinic Tides: Theoretical Modeling and Observational Evidence, 350 pp., Cambridge Univ. Press, New York

Voronovich, A. G. (2003), Strong solitary internal waves in a 2.5-layer model, J. Fluid Mech., 474, 85-94.

K. Hutter, Institute of Mechanics, Darmstadt University of Technology, Hochschulstrasse 1, Darmstadt D-64289, Germany. (hutter@mechanik. tu-darmstadt.de)

L. Ostrovsky, Zel Technologies/NOAA ETL, Boulder, CO 80303, USA. (lev.a.ostrovsky@etl.noaa.gov)

V. Vlasenko, School of Earth, Ocean and Environmental Sciences, University of Plymouth, Drake Circus, PSQ A504, Plymouth, PL4 8AA, UK. (vvlasenko@plymouth.ac.uk) 